\title{
Yeast ecology of vineyards within Marsala wine area (western Sicily) in two consecutive vintages and selection of autochthonous Saccharomyces cerevisiae strains
}

\author{
Luca Settanni, Ciro Sannino, Nicola Francesca, Rosa Guarcello, and Giancarlo Moschetti*
}

DEMETRA Department, University of Palermo, Viale delle Scienze 4, 90128 Palermo, Italy

\author{
Received 7 June 2012; accepted 17 July 2012
}

Available online 9 August 2012

\begin{abstract}
In this work, the yeast ecology associated with the spontaneous fermentation of Grillo cultivar grapes from 10 vineyards was analyzed from grape harvest till complete consumption of must sugars. The microbiological investigation started with the plate count onto two culture media to distinguish total yeasts (TY) and presumptive Saccharomyces (PS). Yeasts were randomly isolated and identified by a combined genotypic approach consisting of restriction fragment length polymorphism (RFLP) of 5.8S rRNA gene and 26S rRNA and sequencing of D1/D2 domain of the 26S rRNA gene, which resulted in the recognition of 14 species belonging to 10 genera. The distribution of the yeasts within the vineyards showed some differences in species composition and concentration levels among 2008 and 2009 vintages. Due to the enological relevance, all Saccharomyces cerevisiae isolates were differentiated applying two genotypic tools (interdelta analysis and microsatellite multiplex PCR of polymorphic microsatellite loci) that recognized 51 strains. Based on the low production of $\mathrm{H}_{2} \mathrm{~S}$, acetic acid and foam, ethanol resistance, growth in presence of high concentrations of potassium metabisulphite (KMBS) and $\mathrm{CuSO}_{4}$ and at low temperatures, 14 strains were selected and used as starter to ferment grape must at $13^{\circ} \mathrm{C}$ and $17^{\circ} \mathrm{C}$ in presence of $100 \mathrm{mg} / \mathrm{L}$ of KMBS. Three strains (CS160, CS165 and CS182) showed optimal technological aptitudes.
\end{abstract}

(c) 2012, The Society for Biotechnology, Japan. All rights reserved.

[Key words: Identification; Enological aptitudes; Saccharomyces cerevisiae; Spontaneous wine fermentation; Yeasts]

Yeasts responsible for the alcoholic fermentation of grape juice into wine are basically distinct in two groups: non-Saccharomyces (NS) species, growing during the first stages of fermentation, and Saccharomyces strains, which become dominant when the ethanol concentration increases.

Since the 80's, starter cultures belonging to the species Saccharomyces cerevisiae are commercially available in order to drive the alcoholic fermentation (1). However, despite the benefits due to the selected yeasts, in terms of effectiveness and ethanol yield, their employment in winemaking is quite controversial. One of the main reason of objection for the routine use of commercial starter yeasts is due to their massive prevalence over the native microflora, with the consequent risk of loss of wine peculiarities (2). Furthermore, the recent growing interest for wines with definite "terroir" characteristics determined a re-discovery of wine fermentation by using indigenous yeasts occurring on grapes and/or in the winery environment (3).

Nowadays, starter cultures selected from autochthonous S. cerevisiae are commonly employed in winemaking to obtain wines with predictable quality and typicality. Although the inoculation of must with selected $S$. cerevisiae is expected to suppress the

\footnotetext{
* Corresponding author. Tel.: +39 091 23896050; fax: +39 0916515531.

E-mail address: giancarlo.moschetti@unipa.it (G. Moschetti).
}

indigenous NS strains, several studies have revealed that NS yeasts can indeed persist during the various stages of wine production driven by pure cultures of $S$. cerevisiae $(4,5)$.

Regarding natural fermentations, Saccharomyces and NS yeasts do not coexist passively. Under these conditions, some enological traits of NS yeasts are not expressed, or may be modulated by S. cerevisiae cultures $(6,7)$. During spontaneous fermentation, NS yeasts contribute to the aroma complexity of wines (8). Some authors reported that these yeasts provide typical aromatic notes that link the wines to the production region $(9,10)$.

The modern trend of wine market is going toward products with given peculiarities. Among special wines, including fortified and non-fortified wines, Marsala produced in the homonymous area of western Sicily is historically known outside Italy since 1773, thanks to the English trader John Woodhouse. Marsala enjoys a "Denominazione di Origine Controllata" (DOC) status that is a recognition of quality (controlled designation of origin). This product requires a base wine for its production and the cultivar Grillo is one of the most cultivated grapevine in Sicily to this purpose.

Keeping in mind that wine production still remains a very traditional process, especially in areas where a long history and typicality of products is felt as an affection to the territory, the objectives of this study were to: examine the qualitative structure and the quantitative development of indigenous yeasts during the 
fermentation of Grillo cultivar (which represents the base wine for Marsala DOC product); to characterize S. cerevisiae isolates at strain level; and to investigate on the enological potential of S. cerevisiae strains.

\section{MATERIALS AND METHODS}

Sample collection Ten vineyards (Table 1) of the "Grillo" variety were sampled for grapes and berries within the Marsala wine production area (Sicily, Italy) during the harvesting of two consecutive vintages (2008 and 2009). The sampling was made in three $100 \mathrm{~m}^{2}$ sub-areas (representing three replicates of the same vineyard) distant approximately 100-300 m from one another. In each vineyard, 15 grapes and $3.0 \mathrm{~kg}$ of grape berries (five grapes and $1 \mathrm{~kg}$ of berries from each sub-area) were randomly collected from undamaged grapes. All samples were then stored at $4{ }^{\circ} \mathrm{C}$ during transport.

Grape samples $(G)$ were placed into sterile plastic bags containing a washing isotonic peptone solution ( $10 \mathrm{~g} / \mathrm{L}$ Bacto Soytone, $2 \mathrm{~mL} / \mathrm{L}$ Tween 80 ) and incubated at $30^{\circ} \mathrm{C}$ for $3 \mathrm{~h}$ to collect the microorganisms hosted on peel surface (11).

Berries were crushed by stomacher (BagMixer ${ }^{\circledR} 400$, Interscience, Saint Nom, France) for $5 \mathrm{~min}$ at the highest speed to obtain must that was transferred into sterile flasks (5 L-volume) and maintained at $17^{\circ} \mathrm{C}$ until total sugar consumption. The samples collected for analysis were: grape must just pressed (M1), must at 1/5 (M2), 3/5 (M3) and 5/5 (M4) of sugar consumption.

Microbiological analysis Cell suspensions recovered from grapes and must samples were serially diluted in Ringer's solution (Sigma-Aldrich, Milan, Italy). Decimal dilutions were spread plated $(0.1 \mathrm{~mL})$ onto Wallerstein laboratory $(\mathrm{WL})$ nutrient agar (Oxoid, Basingstoke, UK), incubated at $28^{\circ} \mathrm{C}$ for $48-72 \mathrm{~h}$, for the counting of total yeasts (TY) and onto modified ethanol sulphite agar (MESA), prepared as reported by Francesca et al. (3), incubated at $28^{\circ} \mathrm{C}$ for $72 \mathrm{~h}$, to detect presumptive Saccharomyces spp. (PS). Both media were supplemented with chloramphenicol $(0.5 \mathrm{~g} / \mathrm{L})$ and biphenyl $(1 \mathrm{~g} / \mathrm{L})$ to inhibit the growth of bacteria and molds, respectively. Analyses were carried out in duplicate.

Statistical analyses were conducted using STATISTICA software (StatSoft Inc., Tulsa, OK, USA). Microbial data were analyzed using a generalized linear model (GLM) including the effects of vineyard ( $\mathrm{V}=$ Guarrato, Lago Preola, Madonna Paradiso, Mazara del Vallo, Mothia, Musciuleo, Pietra Rinosa, Pispisia, Tre Fontane and Triglia Scaletta), year $(Y=2008,2009)$ and sample type $(S=G, M 1-M 4)$ and all their interactions $\left(\mathrm{V}^{*} \mathrm{Y}^{*} \mathrm{~S}\right)$; the Student " $t$ " test was used for mean comparison. The post-hoc Tukey method was applied for pairwise comparison. Significance level was $P<0.05$.

Yeast isolation and identification Yeasts were isolated from both growth media used for counts. Three colonies per morphology were collected from the differential medium WL, while 10 colonies were randomly picked up from MESA. All isolates were purified to homogeneity after several sub-culturing steps onto WL and at least two isolates (from each sample) sharing the same morphology were subjected to the genetic characterization.

The DNA extraction was performed using the InstaGene Matrix kit (Bio-Rad Laboratories, Hercules, CA, USA) according to the manufacturer's instructions.

In order to perform a first differentiation of yeasts, all selected isolates were analyzed by restriction fragment length polymorphism (RFLP) of the region spanning the internal transcribed spacers (ITS1 and ITS2) and the 5.8S rRNA gene. The DNA fragments were amplified and digested as described by Esteve-Zarzoso et al. (12). Gels were stained with SYBR ${ }^{\circledR}$ safe DNA gel stain (Invitrogen, Milan, Italy), visualized by UV transilluminator and acquired by Gel Doc 1000 Video Gel Documentation System (BioRad, Richmond, USA). Standard DNA ladders were $1 \mathrm{~kb}$ Plus DNA Ladder (Invitrogen) and GeneRuler 50 pb DNA Ladder (MBI Fermentas). Five isolates representative of each group were subjected to an additional enzymatic restriction targeting the 26 rRNA gene following the methodology reported by Baleiras-Couto et al. (13). One isolate per group was further processed by sequencing the D1/D2 region of the 26S rRNA gene and/or 5.8S-ITS rRNA region to confirm the preliminary identification obtained by RFLP analysis (14). DNA sequencing reactions were performed at Primmbiotech S.r.l. (Milan, Italy). The identities of the sequences were determined by BlastN search against the NCBI non-redundant sequence database located at http://www.ncbi.nlm.nih.gov.

Strain typing of $\boldsymbol{S}$. cerevisiae isolates Intraspecific characterization of the isolates belonging to $S$. cerevisiae species was carried out through two techniques: interdelta analysis with primers delta 12 and delta 21 (15) and microsatellite multiplex PCR based on the analysis of polymorphic microsatellite loci named SC8132X, YOR267C and SCPTSY7 (16). The PCR products were analyzed on agarose gel $2.0 \%(\mathrm{w} / \mathrm{v})$ in $1 \times$ TBE buffer and visualized as above reported.

Technological characterization of $\boldsymbol{S}$. cerevisiae strains All strains belonging to the species $S$. cerevisiae were evaluated for their potential in winemaking. The ability to produce $\mathrm{H}_{2} \mathrm{~S}$ was tested using a qualitative method performed on Bismuth Sulphite Glucose Glycerin Yeast extract (BiGGY) agar (Oxoid) (17). $\mathrm{H}_{2} \mathrm{~S}$ was estimated by colony blackening after 3 days of incubation at $28^{\circ} \mathrm{C}$. A five-level scale was used for color evaluation: $0=$ white, $1=$ beige, $2=$ light brown, $3=$ brown, $4=$ dark brown, 5 = black. The resistance to various levels of ethanol (from 12 to $16 \% \mathrm{v} / \mathrm{v}$ ) and potassium metabisulphite (KMBS) (from 50 to $300 \mathrm{mg} / \mathrm{L}$ ) were determined onto MESA. S. cerevisiae GR1 (3) and NF213, belonging to the culture collection of DEMETRA Department (University of Palermo, Italy), producing low amount of $\mathrm{H}_{2} \mathrm{~S}$ and resistant to high levels of KMBS and ethanol were used as control strains. Copper tolerance was evaluated as the ability of a strain to grow in presence of different concentration $(50,100,150,200,250,300,350,400,450$ and $500 \mu \mathrm{mol} / \mathrm{L})$ of $\mathrm{CuSO}_{4}$ (18). The strains characterized by high production levels of acetic acid were indicated by the halo produced around colonies onto $\mathrm{CaCO}_{3}$ agar plates after 7-day incubation at $25^{\circ} \mathrm{C}$ (19). S. cerevisiae GR1 was used as negative control, while Hanseniaspora uvarum TLM14 (DEMETRA culture collection) as positive control. The

TABLE 1. Microbial loads ${ }^{\mathrm{a}}$ of samples collected from Grillo vineyards and micro fermentations.

\begin{tabular}{|c|c|c|c|c|c|c|c|c|c|c|}
\hline \multirow[t]{2}{*}{ Samples } & \multicolumn{10}{|c|}{ Vineyards } \\
\hline & $\begin{array}{c}\text { Guarrato } \\
37^{\circ} 56^{\prime} \\
N-12^{\circ} 32^{\prime} \mathrm{E}\end{array}$ & $\begin{array}{c}\text { Lago Preola } \\
37^{\circ} 36^{\prime} \\
\mathrm{N}-12^{\circ} 38^{\prime} \mathrm{E}\end{array}$ & $\begin{array}{c}\text { Madonna } \\
\text { Paradiso } \\
37^{\circ} 40^{\prime} \mathrm{N}-12^{\circ} 36^{\prime} \mathrm{E}\end{array}$ & $\begin{array}{c}\text { Mazara del } \\
\text { Vallo } 37^{\circ} 41^{\prime} \\
\mathrm{N}-12^{\circ} 35^{\prime} \mathrm{E}\end{array}$ & $\begin{array}{c}\text { Mothia } 37^{\circ} \\
52^{\prime} \mathrm{N}-12^{\circ} \\
28^{\prime} \mathrm{E}\end{array}$ & $\begin{array}{c}\text { Musciuleo } \\
37^{\circ} 52^{\prime} \\
\mathrm{N}-12^{\circ} 34^{\prime} \mathrm{E}\end{array}$ & $\begin{array}{c}\text { Pietra Rinosa } \\
37^{\circ} 52^{\prime} \\
N-12^{\circ} 43^{\prime} \mathrm{E}\end{array}$ & $\begin{array}{l}\text { Pispisia } 37^{\circ} 50^{\prime} \\
\quad \mathrm{N}-12^{\circ} 29^{\prime} \mathrm{E}\end{array}$ & $\begin{array}{c}\text { Tre Fontane } \\
37^{\circ} 34^{\prime} \mathrm{N}-12^{\circ} \\
42^{\prime} \mathrm{E}\end{array}$ & $\begin{array}{c}\text { Triglia Scaletta } \\
37^{\circ} 43^{\prime} \mathrm{N}-12^{\circ} 31^{\prime} \mathrm{E}\end{array}$ \\
\hline \multicolumn{11}{|l|}{ TY (2008) } \\
\hline G & $6.0 \pm 0.3$ & $5.13 \pm 0.3$ & $3.54 \pm 0.6$ & $4.98 \pm 0.7$ & $6.92 \pm 0.3$ & $6.39 \pm 0.2$ & $5.12 \pm 0.5$ & $5.65 \pm 0.2$ & $6.41 \pm 0.2$ & $6.84 \pm 0.5$ \\
\hline M1 & $6.25 \pm 0.3$ & $5.60 \pm 0.4$ & $3.27 \pm 0.3$ & $5.98 \pm 0.4$ & $6.78 \pm 0.4$ & $6.64 \pm 0.3$ & $5.36 \pm 0.4$ & $6.67 \pm 0.4$ & $6.81 \pm 0.3$ & $6.99 \pm 0.2$ \\
\hline M2 & $7.38 \pm 0.4$ & $6.87 \pm 0.8$ & $7.15 \pm 0.2$ & $7.08 \pm 0.2$ & $8.28 \pm 0.3$ & $5.99 \pm 0.5$ & $5.77 \pm 0.4$ & $8.24 \pm 0.4$ & $7.17 \pm 0.0$ & $7.46 \pm 0.2$ \\
\hline M3 & $8.15 \pm 0.1$ & $8.05 \pm 0.4$ & $7.91 \pm 0.7$ & $7.96 \pm 0.2$ & $7.89 \pm 0.4$ & $4.93 \pm 0.4$ & $4.13 \pm 0.2$ & $7.84 \pm 0.5$ & $6.55 \pm 0.5$ & $8.01 \pm 0.3$ \\
\hline M4 & $8.09 \pm 0.4$ & $4.79 \pm 0.4$ & $4.42 \pm 0.4$ & $8.09 \pm 0.5$ & $7.98 \pm 0.6$ & $2.93 \pm 0.1$ & $1.39 \pm 0.5$ & $7.54 \pm 0.6$ & $4.16 \pm 0.1$ & $7.21 \pm 0.5$ \\
\hline \multicolumn{11}{|l|}{ PS (2008) } \\
\hline G & $2.47 \pm 0.2$ & n.d. & n.d. & n.d. & n.d. & n.d. & n.d. & n.d. & n.d. & n.d. \\
\hline M1 & $3.06 \pm 0.1$ & n.d. & n.d. & n.d. & $3.92 \pm 0.1$ & n.d. & n.d. & n.d. & n.d. & n.d. \\
\hline M2 & $6.20 \pm 0.1$ & $3.56 \pm 0.2$ & $3.12 \pm 0.2$ & $5.88 \pm 0.7$ & $7.14 \pm 0.2$ & $5.08 \pm 0.1$ & $3.29 \pm 0.4$ & $6.5 \pm 0.8$ & $5.23 \pm 0.3$ & $5.71 \pm 0.6$ \\
\hline M3 & $8.16 \pm 0.8$ & $4.14 \pm 0.0$ & $4.62 \pm 0.5$ & $6.46 \pm 0.1$ & $6.76 \pm 0.3$ & $2.24 \pm 0.4$ & $2.94 \pm 0.3$ & $7.16 \pm 0.0$ & $5.02 \pm 0.1$ & $7.50 \pm 0.7$ \\
\hline M4 & $7.36 \pm 0.5$ & $3.81 \pm 0.2$ & $3.44 \pm 0.3$ & $7.48 \pm 0.3$ & $7.02 \pm 0.7$ & $1.0 \pm 0.0$ & n.d. & $7.37 \pm 0.5$ & $2.02 \pm 0.1$ & $6.72 \pm 0.5$ \\
\hline \multicolumn{11}{|c|}{ TY (2009) } \\
\hline G & $5.56 \pm 0.4$ & $5.79 \pm 0.2$ & $5.93 \pm 0.8$ & $6.08 \pm 0.2$ & $4.07 \pm 0.2$ & $4.01 \pm 0.3$ & $5.77 \pm 0.5$ & $4.29 \pm 0.3$ & $4.36 \pm 0.4$ & $3.16 \pm 0.6$ \\
\hline M1 & $5.25 \pm 0.8$ & $6.30 \pm 0.3$ & $6.09 \pm 0.6$ & $6.6 \pm 0.3$ & $5.0 \pm 0.3$ & $5.54 \pm 0.4$ & $5.25 \pm 0.4$ & $5.03 \pm 0.5$ & $5.29 \pm 0.4$ & $3.98 \pm 0.5$ \\
\hline M2 & $7.39 \pm 0.9$ & $7.20 \pm 0.3$ & $8.25 \pm 0.3$ & $7.76 \pm 0.2$ & $7.97 \pm 0.4$ & $5.91 \pm 0.7$ & $7.20 \pm 0.4$ & $7.81 \pm 0.3$ & $8.09 \pm 0.2$ & $5.84 \pm 0.2$ \\
\hline M3 & $7.59 \pm 0.4$ & $7.27 \pm 0.5$ & $8.78 \pm 0.7$ & $7.38 \pm 0.4$ & $7.83 \pm 0.6$ & $4.26 \pm 0.5$ & $7.09 \pm 0.2$ & $7.55 \pm 0.2$ & $7.85 \pm 0.6$ & $6.77 \pm 0.4$ \\
\hline M4 & $7.27 \pm 0.4$ & $8.16 \pm 0.6$ & $8.17 \pm 0.1$ & $7.53 \pm 0.1$ & $7.97 \pm 0.5$ & $1.86 \pm 0.4$ & $5.95 \pm 0.7$ & $7.66 \pm 0.3$ & $7.54 \pm 0.3$ & $6.27 \pm 0.7$ \\
\hline \multicolumn{11}{|l|}{ PS (2009) } \\
\hline G & n.d. & n.d. & n.d. & n.d. & n.d. & n.d. & n.d. & n.d. & $1.94 \pm 0.5$ & n.d. \\
\hline M1 & n.d. & $2.13 \pm 0.7$ & $1.84 \pm 0.4$ & n.d. & $2.66 \pm 0.1$ & n.d. & n.d. & $3.07 \pm 0.1$ & $3.44 \pm 0.6$ & $2.03 \pm 0.1$ \\
\hline M2 & $5.47 \pm 0.3$ & $5.47 \pm 0.1$ & $7.76 \pm 0.6$ & $2.87 \pm 0.3$ & $5.64 \pm 0.5$ & $3.85 \pm 0.9$ & $6.30 \pm 0.4$ & $5.22 \pm 0.3$ & $5.12 \pm 0.2$ & $4.15 \pm 0.1$ \\
\hline M3 & $7.4 \pm 0.0$ & $7.21 \pm 0.5$ & $8.77 \pm 0.4$ & $5.10 \pm 0.1$ & $6.60 \pm 0.8$ & $3.12 \pm 0.2$ & $5.85 \pm 0.3$ & $7.54 \pm 0.7$ & $7.22 \pm 0.3$ & $5.92 \pm 0.6$ \\
\hline M4 & $7.17 \pm 0.3$ & $7.04 \pm 0.0$ & $6.97 \pm 0.2$ & $6.90 \pm 0.9$ & $6.89 \pm 0.6$ & n.d. & $5.62 \pm 0.9$ & $7.07 \pm 0.1$ & $6.97 \pm 0.1$ & $6.16 \pm 0.1$ \\
\hline
\end{tabular}

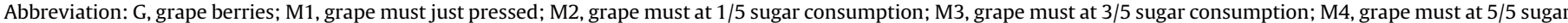
consumption; TY, total yeasts on WL nutrient agar; PS, presumptive Saccharomyces on MESA; n.d., not determined.

${ }^{a} \mathrm{Log}$ CFU/g for grape berries; Log CFU/mL for must samples. 
growth at low temperatures was determined in Yeast Extract Peptone Dextrose (YPD) broth at $13^{\circ} \mathrm{C}$ and $17{ }^{\circ} \mathrm{C}$ for 5 days. Growth patterns were examined through visual inspection of samples through a light microscope (Carl Zeiss Ltd.) (20). Foam production was examined according to Regodón et al. (21).

The strains selected for must fermentation were also evaluated for their enzymatic activities: $\beta$-glucosidase activity was tested in presence of arbutin, esculin, 4-methylumbelliferil $\beta$-D-glucopyranoside (MUG) and 4-nitrophenyl $\beta$-D-glucopyranoside ( $p$-NPG) (22); proteolytic activity was assayed as reported by Bilinsky et al. (23).

All analyses were carried out in triplicate.

Micro fermentations The strains showing the best technological performances (low production of $\mathrm{H}_{2} \mathrm{~S}$ and acetic acid, resistance to ethanol, $\mathrm{KMBS}_{\text {and }} \mathrm{CuSO}_{4}$, ability to grow at low temperatures, growth in suspended form and low foam production) were evaluated for their ability to ferment a grape must. Broth cultures in the stationary phase were washed twice in Ringer's solution and inoculated in $1 \mathrm{~L}$ of

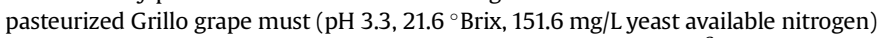
added with KMBS $(100 \mathrm{mg} / \mathrm{L})$ at a final concentration of about $10^{6} \mathrm{CFU} / \mathrm{mL}$. Micro fermentations were carried out at $13{ }^{\circ} \mathrm{C}$ and $17{ }^{\circ} \mathrm{C}$. In order to allow $\mathrm{CO}_{2}$ removal, the flasks were plugged with a Müller valve containing sulfuric acid (24) and the weight loss was monitored until the daily decrease was lower than $0.01 \mathrm{~g}$ (end of fermentation process). According to Ciani and Maccarelli (25), the fermentation power (FP) was evaluated as ethanol amount $(\% \mathrm{v} / \mathrm{v})$ produced at the end of the process, the fermentation rate (FR) was calculated as $\mathrm{CO}_{2}$ daily produced and the fermentation purity $(\mathrm{FPu})$ was calculated as acetic acid $(\mathrm{g} / \mathrm{L})$ per ethanol $(\% \mathrm{v} / \mathrm{v})$ produced at the end of micro fermentation. Two control micro fermentations were inoculated with S. cerevisiae GR1 and F1 (DEMETRA culture collection). At the end of fermentation, the wines were analyzed for residual sugar, acetic acid and glycerol content following the standard methods of the Organization of Vine and Wine (26).

\section{RESULTS}

Microbiological analysis The viable counts of TY and PS populations investigated in this study are reported in Table 1 . TY counts on the grape surface were in the range 3.54-6.92 and 3.16-6.08 Log CFU/g in vintage 2008 and 2009, respectively. On average, higher levels of TY were observed on grapes collected in $2008(P<0.05)$, that were above $6 \mathrm{Log}$ CFU/g for Mothia, Musciuleo, Tre Fontane and Triglia Scaletta vineyards. Data recovered from MESA showed that, except samples from Guarrato vineyard in the vintage 2008 and Tre Fontane vineyard in the vintage 2009, grapes did not host yeasts ascribable to PS group at detectable levels.

The yeast populations analyzed at different steps during sugar consumption were also monitored. TY load of M1 samples were higher than that detected on the corresponding grapes $(P<0.05)$. Regarding PS populations, the concentrations found for M1 samples from Guarrato 2008 and Tre Fontane 2009 were higher $(P<0.05)$ than those found in $\mathrm{G}$ samples and detectable levels were registered in six other M1 samples. During fermentation, both TY and PS counts increased significantly $(P<0.05)$; although often M3 samples showed higher levels than M4, not always the highest concentrations were displayed by M3 samples, since in some cases it was registered for M4 or M2 samples.

In general, the effect of vineyard, year and sample type was found to significantly $(P<0.001)$ affect count data of PS, while for TY the concentration levels were affected by vineyard $(P<0.001)$ and sample type $(P<0.001)$, but not by year. The combination of the three independent variables $\left(\mathrm{V}^{*} \mathrm{Y}^{*} \mathrm{~S}\right)$ significantly affected both PS and TY counts.

Isolation and identification of yeasts A total of 1144 colonies from WL and 987 from MESA were isolated, purified to homogeneity and separated on the basis of appearance of colony morphology on WL. At least two cultures from each sample were morphologically selected obtaining 1021 isolates (614 from WL and 407 from MESA) which were subjected to molecular identification. After restriction analysis of 5.8S-ITS region and 26S rRNA gene, the isolates were clustered in 14 groups (Table 2): three of these groups (X, XI and XIII) were directly identified by comparison of restriction bands with those available in literature $(12,27,28)$. These patterns corresponded to Lachancea thermotolerans, Metschnikowia pulcherrima and S. cerevisiae species. Eleven groups could not be identified by RFLP analysis, then the identification at species level

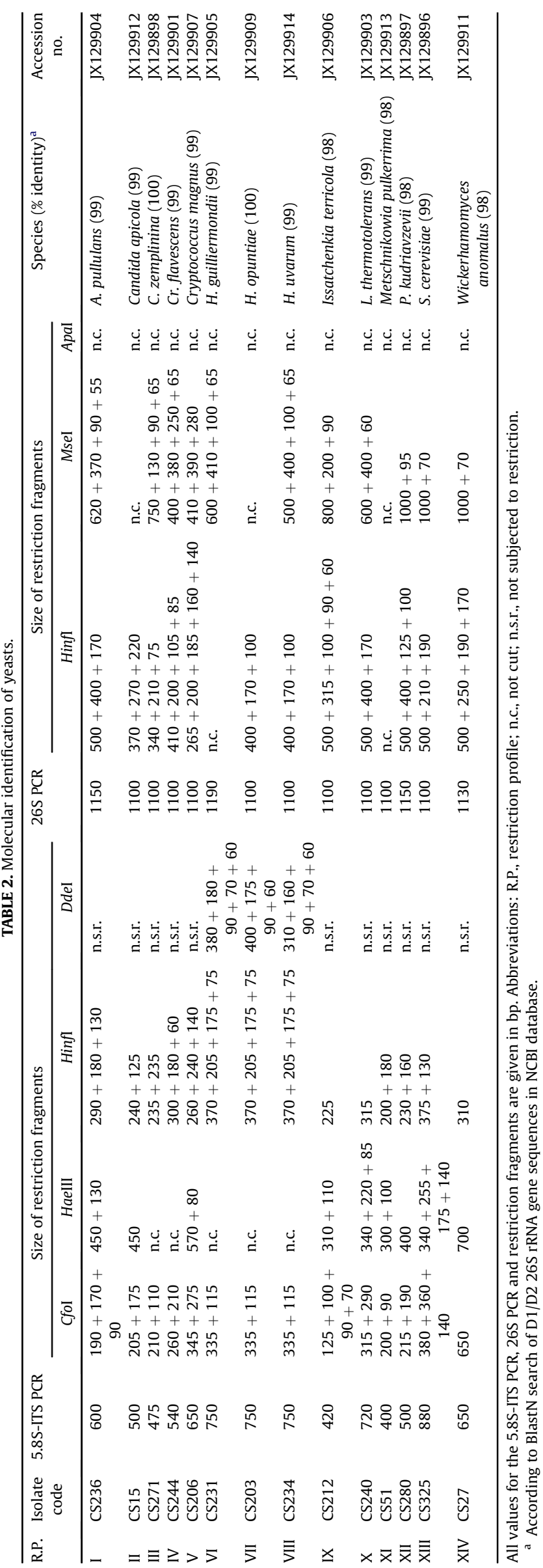


TABLE 3. Geographical and annual distribution ${ }^{\mathrm{a}}$ of yeast species during spontaneous fermentations.

\begin{tabular}{|c|c|c|c|c|c|c|c|c|c|c|}
\hline \multirow[t]{2}{*}{ Species } & \multicolumn{10}{|c|}{ Vineyards } \\
\hline & Guarrato & Lago Preola & Madonna Paradiso & Mazara del Vallo & Mothia & Musciuleo & Pietra Rinosa & Pispisia & Tre Fontane & Triglia Scaletta \\
\hline \multicolumn{11}{|l|}{2008} \\
\hline A. pullulans & & & & $G\left(4^{b}\right)$ & & & $\mathrm{G}\left(5^{\mathrm{b}}\right) \mathrm{M} 1\left(5^{\mathrm{b}}\right)$ & & & \\
\hline C. apicola & & & & & & & & & $\mathrm{G}\left(6^{\mathrm{b}}\right) \mathrm{M} 1\left(6^{\mathrm{b}}\right)$ & \\
\hline C. zemplinina & & & & $\operatorname{M} 1\left(5^{b}\right) \operatorname{M} 2\left(7^{b}\right)$ & & & $\mathrm{M} 2\left(5^{\mathrm{b}}\right)$ & & & \\
\hline Cr. flavescens & & & & & & & & & & \\
\hline Cr. magnus & & & & & & & & & & \\
\hline H. guilliermondii & & & & & & & & & & \\
\hline H. opuntiae & & & & & & & $\mathrm{M} 2\left(5^{\mathrm{b}}\right) \mathrm{M} 3\left(4^{\mathrm{b}}\right)$ & & & \\
\hline H. uvarum & $\begin{array}{l}\mathrm{G}\left(6^{\mathrm{b}}, 2^{\mathrm{c}}\right) \mathrm{M} 1\left(6^{\mathrm{b}}, 4^{\mathrm{c}}\right) \\
\quad \mathrm{M} 2\left(7^{\mathrm{b}, \mathrm{c}}\right) \mathrm{M} 3\left(8^{\mathrm{b}}, 7^{\mathrm{c}}\right)\end{array}$ & $\begin{array}{l}\text { M2( }\left(6^{b}\right) \text { M3 } 3\left(8^{b}, 4^{c}\right) \\
\text { M4 } 4\left(8^{b}, 3^{c}\right)\end{array}$ & $\begin{array}{l}\text { M2 }\left(7^{b}, 3^{c}\right) \\
\text { M3 }\left(7^{b}, 4^{c}\right) \\
\text { M4 }\left(3^{c}\right)\end{array}$ & $\begin{array}{r}\mathrm{M} 2\left(7^{\mathrm{b}}, 5^{\mathrm{c}}\right) \\
\mathrm{M} 3\left(7^{\mathrm{b}}\right)\end{array}$ & $\begin{array}{l}G\left(6^{b}\right) M 1\left(6^{b}, 3^{c}\right) \\
\text { M2 }\left(8^{b}, 7^{c}\right) \\
\text { M3 }\left(7^{b}, 6^{c}\right)\end{array}$ & $\begin{array}{l}\text { M1 }\left(6^{b}\right) \text { M2( }\left(6^{b}, 5^{c}\right) \\
\text { M3 }\left(4^{\mathrm{b}}, 2^{c}\right) \\
\text { M4 }\left(2^{\mathrm{b}}, 1^{\mathrm{c}}\right)\end{array}$ & & $\mathrm{M} 2\left(8^{\mathrm{b}}, 6^{\mathrm{c}}\right) \mathrm{M} 3\left(7^{\mathrm{b}}\right)$ & $\begin{array}{l}\text { M1 }\left(6^{b}\right) \\
\text { M2( }\left(7^{b}, 5^{c}\right)\end{array}$ & $\begin{array}{l}\mathrm{G}\left(6^{\mathrm{b}}\right) \mathrm{M} 1\left(6^{\mathrm{b}}\right) \\
\mathrm{M} 2\left(7^{\mathrm{b}}, 5^{\mathrm{c}}\right)\end{array}$ \\
\hline I. terricola & & & & & & & & & & \\
\hline L. thermotolerans & $\begin{array}{l}G\left(2^{\mathrm{c}}\right) \mathrm{M} 1\left(6^{\mathrm{b}}, 3^{\mathrm{c}}\right) \\
\mathrm{M} 2\left(7^{\mathrm{b}}\right)\end{array}$ & & & & & & $\mathrm{M} 2\left(5^{\mathrm{b}}, 3^{\mathrm{c}}\right) \mathrm{M} 3\left(4^{\mathrm{b}}\right)$ & & & \\
\hline M. pulcherrima & $\mathrm{G}\left(6^{\mathrm{b}}\right) \mathrm{M} 1\left(6^{\mathrm{b}}\right)$ & & $\begin{array}{l}G\left(3^{b}\right) M 1\left(3^{b}\right) \\
M 2\left(7^{b}\right)\end{array}$ & $\mathrm{M} 1\left(5^{\mathrm{b}}\right)$ & & & & $\begin{array}{l}G\left(5^{\mathrm{b}}\right) \mathrm{M} 1\left(6^{\mathrm{b}}\right) \\
\quad \mathrm{M} 2\left(8^{\mathrm{b}}\right) \mathrm{M} 3\left(7^{\mathrm{b}}\right)\end{array}$ & $G\left(6^{b}\right) M 1\left(6^{b}\right)$ & \\
\hline P. kudriavzevii & & & & & & & $\begin{array}{l}\operatorname{M} 2\left(3^{\mathrm{c}}\right) \operatorname{M} 3\left(4^{\mathrm{b}}, 2^{\mathrm{c}}\right) \\
\mathrm{M} 4\left(1^{\mathrm{b}}\right)\end{array}$ & & $\begin{array}{l}\text { M3 }\left(6^{\mathrm{b}}, 5^{\mathrm{c}}\right) \\
\text { M4 }\left(4^{\mathrm{b}}, 2^{\mathrm{c}}\right)\end{array}$ & \\
\hline S. cerevisiae & $\begin{array}{c}\mathrm{M} 2\left(6^{\mathrm{c}}\right) \mathrm{M} 3\left(8^{\mathrm{b}, \mathrm{c}}\right) \\
\mathrm{M} 4\left(8^{\mathrm{b}}, 7^{\mathrm{c}}\right)\end{array}$ & & & $\begin{array}{l}\text { M3 }\left(7^{b}, 6^{c}\right) \\
\text { M4 }\left(8^{b}, 7^{c}\right)\end{array}$ & $\begin{array}{c}M 1\left(6^{\mathrm{b}}, 3^{\mathrm{c}}\right) \\
\mathrm{M} 3\left(6^{\mathrm{c}}\right) \\
\mathrm{M} 4\left(7^{\mathrm{b}, \mathrm{c}}\right)\end{array}$ & & & $\mathrm{M} 3\left(7^{\mathrm{b}, \mathrm{c}}\right) \mathrm{M} 4\left(7^{\mathrm{b}, \mathrm{c}}\right)$ & & $\begin{array}{l}\text { M2 }\left(7^{\mathrm{b}}, 5^{\mathrm{c}}\right) \\
\text { M3 } 3\left(8^{\mathrm{b}}, 7^{\mathrm{c}}\right) \\
\text { M4 }\left(7^{\mathrm{b}}, 6^{\mathrm{c}}\right)\end{array}$ \\
\hline W. anomalus & & & & & & $\mathrm{G}\left(6^{\mathrm{b}}\right) \mathrm{M} 1\left(6^{\mathrm{b}}\right)$ & & & & \\
\hline 2009 & & & & & & & & & & \\
\hline $\begin{array}{l}\text { A. pullulans } \\
\text { C. apicola }\end{array}$ & & $G\left(5^{b}\right) M 1\left(6^{b}\right)$ & & $G\left(6^{b}\right) M 1\left(6^{b}\right)$ & & $G\left(4^{b}\right)$ & $G\left(5^{b}\right) M 1\left(5^{b}\right)$ & $G\left(4^{b}\right) M 1\left(5^{b}\right)$ & $\mathrm{G}\left(4^{\mathrm{b}}\right) \mathrm{M} 1\left(5^{\mathrm{b}}\right)$ & \\
\hline C. zemplinina & & $\begin{array}{l}\mathrm{M} 2\left(7^{\mathrm{b}}, 5^{\mathrm{c}}\right) \mathrm{M} 3\left(7^{\mathrm{b}, \mathrm{c}}\right) \\
\mathrm{M} 4\left(8^{\mathrm{b}}, 7^{\mathrm{c}}\right)\end{array}$ & & & $\begin{array}{l}\operatorname{M} 1\left(5^{b}, 2^{c}\right) \\
\quad \operatorname{M}\left(7^{b}, 5^{c}\right)\end{array}$ & & $\mathrm{M} 2\left(7^{\mathrm{b}}, 6^{\mathrm{c}}\right) \mathrm{M} 3\left(5^{\mathrm{b}, \mathrm{c}}\right)$ & & $\operatorname{M} 1\left(5^{\mathrm{b}}\right)$ & $\begin{array}{c}G\left(3^{b}\right) M 1\left(3^{b}, 2^{c}\right) \\
M 2\left(5^{b}, 4^{c}\right) \\
M 3\left(6^{b}, 5^{c}\right) \\
M 4\left(6^{b, c}\right)\end{array}$ \\
\hline Cr. flavescens & & & & & $G\left(4^{b}\right)$ & & & & & \\
\hline Cr. magnus & & $G\left(5^{b}\right) M 1\left(6^{b}\right)$ & & & & & & & & \\
\hline H. guilliermondii & & $\mathrm{M} 2\left(7^{\mathrm{b}}\right)$ & $G\left(5^{b}\right)$ & & & & & & $\begin{array}{c}G\left(4^{b}\right) M 1\left(5^{b}\right) \\
M 3\left(7^{b}, 6^{c}\right) \\
M 4\left(7^{b}, 6^{c}\right)\end{array}$ & $\mathrm{G}\left(3^{\mathrm{b}}\right) \mathrm{M} 1\left(3^{\mathrm{b}}\right)$ \\
\hline H. opuntiae & $\begin{array}{l}\mathrm{G}\left(5^{\mathrm{b}}\right) \mathrm{M} 1\left(5^{\mathrm{b}}\right) \mathrm{M} 2\left(7^{\mathrm{b}}\right) \\
\mathrm{M} 3\left(7^{\mathrm{b}}\right)\end{array}$ & $\operatorname{M} 1\left(6^{\mathrm{b}}\right)$ & $\mathrm{M} 1\left(6^{\mathrm{b}}\right) \mathrm{M} 2\left(8^{\mathrm{b}}\right)$ & $\operatorname{M} 2\left(7^{b}\right) M 3\left(7^{b}\right)$ & & & $\begin{array}{l}\mathrm{G}\left(5^{\mathrm{b}}\right) \mathrm{M} 1\left(5^{\mathrm{b}}\right) \\
\mathrm{M} 2\left(7^{\mathrm{b}}\right)\end{array}$ & $\begin{array}{l}\operatorname{M1} 1\left(5^{\mathrm{b}}, 3^{\mathrm{M}}\right) \\
\text { M2( }\left(7^{\mathrm{b}}\right) \operatorname{M} 3\left(7^{\mathrm{b}}\right)\end{array}$ & $\operatorname{M} 2\left(8^{\mathrm{b}}\right) \mathrm{M} 3\left(7^{\mathrm{b}}\right)$ & \\
\hline H. uvarum & $\mathrm{M} 3\left(7^{\mathrm{b}, \mathrm{c}}\right) \mathrm{M} 4\left(7^{\mathrm{b}, \mathrm{c}}\right)$ & $\begin{array}{l}\mathrm{G}\left(5^{\mathrm{b}}\right) \mathrm{M} 1\left(2^{\mathrm{b}, \mathrm{c}}\right) \\
\quad \mathrm{M} 2\left(7^{\mathrm{b}}, 5^{\mathrm{c}}\right) \mathrm{M} 3\left(7^{\mathrm{b}, \mathrm{c}}\right) \\
\mathrm{M} 4\left(8^{\mathrm{b}}, 7^{\mathrm{c}}\right)\end{array}$ & $\mathrm{M} 2\left(8^{\mathrm{b}}, 7^{\mathrm{c}}\right) \mathrm{M} 3\left(8^{\mathrm{b}}\right)$ & $\mathrm{M} 2\left(7^{\mathrm{b}}\right) \mathrm{M} 3\left(7^{\mathrm{b}}\right)$ & $\begin{array}{l}\operatorname{M1} 1\left(5^{b}\right) \operatorname{M} 2\left(7^{b}, 5^{c}\right) \\
\quad M 3\left(7^{b}\right)\end{array}$ & $\begin{array}{l}\operatorname{M} 2\left(3^{\mathrm{c}}\right) \mathrm{M} 3\left(4^{\mathrm{b}}, 3^{\mathrm{c}}\right) \\
\quad \mathrm{M} 4\left(1^{\mathrm{b}}\right)\end{array}$ & & & $\operatorname{M} 1\left(5^{b}, 3^{c}\right)$ & \\
\hline $\begin{array}{l}\text { I. terricola } \\
\text { L. thermotolerans }\end{array}$ & & & $G\left(5^{b}\right) M 1\left(6^{b}\right)$ & & & & $\operatorname{M} 1\left(5^{\mathrm{b}}\right) \mathrm{M} 4\left(6^{\mathrm{b}} 4^{\mathrm{c}}\right)$ & & $\mathrm{G}\left(5^{\mathrm{b}}\right) \mathrm{M} 2\left(5^{\mathrm{b}}\right)$ & \\
\hline M. pulcherrima & $\mathrm{M} 1\left(5^{\mathrm{b}}\right) \mathrm{M} 2\left(7^{\mathrm{b}}\right)$ & $\mathrm{M} 1\left(5^{\mathrm{b}}\right) \mathrm{M} 2\left(6^{\mathrm{b}}\right)$ & $\operatorname{M} 1\left(6^{\mathrm{b}}\right)$ & $\mathrm{M} 1\left(6^{\mathrm{b}}\right)$ & & $\operatorname{M} 1\left(5^{b}\right) M 2\left(5^{b}\right)$ & & $\mathrm{M} 2\left(7^{\mathrm{b}}\right) \mathrm{M} 3\left(7^{\mathrm{b}}\right)$ & $\mathrm{M} 1\left(5^{\mathrm{b}}\right)$ & \\
\hline P. kudriavzevii & $\mathrm{M} 4\left(7^{\mathrm{b}, \mathrm{c}}\right)$ & $\begin{array}{l}\mathrm{M} 1\left(6^{b}\right) \operatorname{M} 2\left(5^{c}\right) \\
M 3\left(7^{b}\right)\end{array}$ & & $\begin{array}{l}\text { M2 }\left(2^{c}\right) \operatorname{M} 3\left(7^{b}\right) \\
M 4\left(7^{b}, 6^{c}\right)\end{array}$ & & & $\mathrm{M} 3\left(7^{\mathrm{b}}, 5^{\mathrm{c}}\right) \mathrm{M} 4\left(5^{\mathrm{b}, \mathrm{c}}\right)$ & & $G\left(1^{c}\right) M 3\left(7^{b}\right)$ & \\
\hline S. cerevisiae & $\mathrm{M} 3\left(7^{\mathrm{b}, \mathrm{c}}\right) \mathrm{M} 4\left(7^{\mathrm{b}, \mathrm{c}}\right)$ & $\mathrm{M} 3\left(7^{\mathrm{b}, \mathrm{c}}\right) \mathrm{M} 4\left(8^{\mathrm{b}, \mathrm{c}}\right)$ & $\mathrm{M} 3\left(8^{\mathrm{b}, \mathrm{c}}\right) \mathrm{M} 4\left(8^{\mathrm{b}, \mathrm{c}}\right)$ & $\begin{array}{l}\text { M3 }\left(7^{b}, 5^{c}\right) \\
M 4\left(7^{b}, 6^{c}\right)\end{array}$ & $\begin{array}{l}\text { M3 }\left(7^{\mathrm{b}}, 6^{\mathrm{c}}\right) \\
\quad \mathrm{M} 4\left(7^{\mathrm{b}}, 6^{\mathrm{c}}\right)\end{array}$ & & & $\begin{array}{l}\mathrm{M} 2\left(7^{\mathrm{b}}, 5^{\mathrm{c}}\right) \mathrm{M} 3\left(7^{\mathrm{b}, \mathrm{c}}\right) \\
\mathrm{M} 4\left(7^{\mathrm{b}, \mathrm{c}}\right)\end{array}$ & $\begin{array}{c}\text { M1 }\left(3^{\mathrm{c}}\right) \mathrm{M} 2\left(5^{\mathrm{c}}\right) \\
\text { M3 }\left(7^{\mathrm{b}^{\mathrm{b}} \mathrm{c}}\right) \\
\mathrm{M} 4\left(7^{\mathrm{b}}, 6^{\mathrm{c}}\right)\end{array}$ & \\
\hline
\end{tabular}

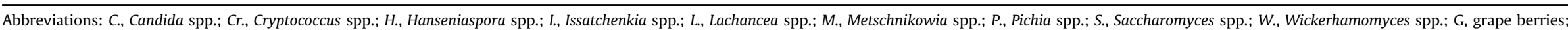
M1, grape must just pressed; M2, grape must at $1 / 5$ sugar consumption; M3, grape must at $3 / 5$ sugar consumption; M4, grape must at 5/5 sugar consumption.

The number reported between brackets refers to the highest concentration (Log cycle) of detection.

Yeast count onto WL nutrient agar.

Yeast count onto MESA. 
was concluded by sequencing of D1/D2 domain of the 26S rRNA gene which was successful for all groups obtained by enzymatic digestions.

Yeast species distribution The distribution of yeast species among vineyards and vintages, as well as their concentration estimated for each sample, are reported in Table 3. H. uvarum, M. pulcherrima and Aureobasidium pullulans were the species most frequently encountered on grapes and musts soon after pressing. In general, the concentration levels detected on WL were higher than those found on MESA. S. cerevisiae was never detected on grapes and twice in M1 (Mothia 2008 and Tre Fontane 2009). The concentration of $S$. cerevisiae was relevant (approximately $10^{6} \mathrm{CFU} /$ $\mathrm{mL}$ ) in M1 from vineyard Mothia in vintage 2008. The samples M2 and M3 were dominated by H. uvarum, S. cerevisiae and Candida zemplinina in both years reaching levels ranging between 6 and 8 orders of magnitude. Hanseniaspora opuntiae was also isolated in several M2 and M3 samples at high concentrations but only in the vintage 2009. At the end of the fermentation process, S. cerevisiae, H. uvarum and Pichia kudriavzevii were detected in several M4 samples of the two consecutive vintages and C. zemplinina only in 2008. Interestingly, in this technological step, the yeast levels found on MESA were comparable or even superimposable with

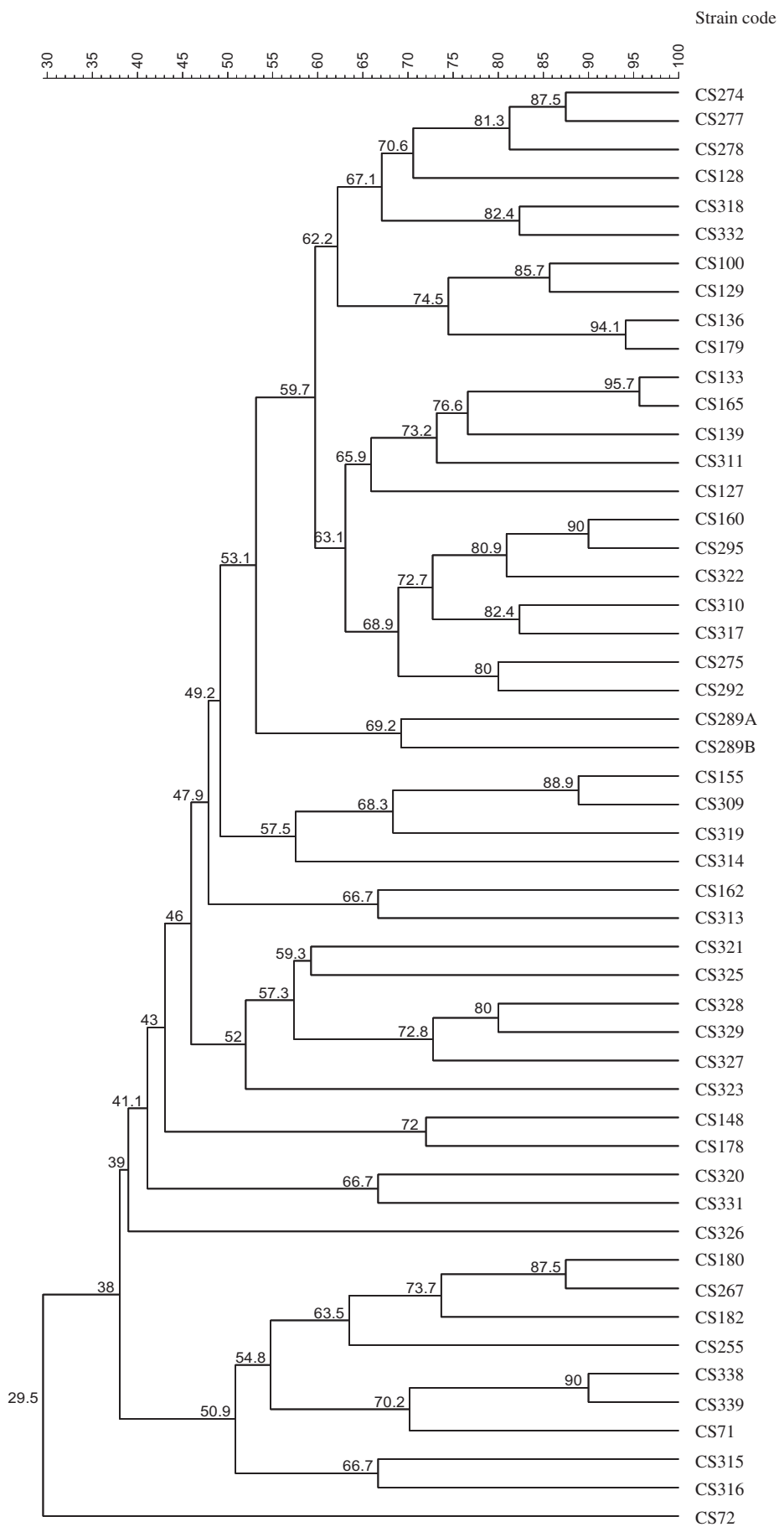

\begin{tabular}{|c|c|}
\hline Vineyard & Year \\
\hline Madonna Paradiso & 2009 \\
\hline Mothia & 2009 \\
\hline Mothia & 2009 \\
\hline Mothia & 2008 \\
\hline Mazara del Vallo & 2009 \\
\hline Pispisia & 2009 \\
\hline Triglia Scaletta & 2008 \\
\hline Mothia & 2008 \\
\hline Mazara del Vallo & 2008 \\
\hline Mazara del Vallo & 2008 \\
\hline Guarrato & 2008 \\
\hline Triglia Scaletta & 2008 \\
\hline Triglia Scaletta & 2008 \\
\hline Tre Fontane & 2009 \\
\hline Mothia & 2008 \\
\hline Pispisia & 2008 \\
\hline Lago Preola & 2009 \\
\hline Mothia & 2009 \\
\hline Tre Fontane & 2009 \\
\hline Mazara del Vallo & 2009 \\
\hline Madonna Paradiso & 2009 \\
\hline Tre Fontane & 2009 \\
\hline Pispisia & 2009 \\
\hline Pispisia & 2009 \\
\hline Guarrato & 2008 \\
\hline Guarrato & 2009 \\
\hline Mazara del Vallo & 2009 \\
\hline Tre Fontane & 2009 \\
\hline Mazara del Vallo & 2008 \\
\hline Tre Fontane & 2009 \\
\hline Mazara del Vallo & 2009 \\
\hline Mothia & 2009 \\
\hline Pispisia & 2009 \\
\hline Pispisia & 2009 \\
\hline Mothia & 2009 \\
\hline Mothia & 2009 \\
\hline Pispisia & 2008 \\
\hline Pispisia & 2008 \\
\hline Mazara del Vallo & 2009 \\
\hline Pispisia & 2009 \\
\hline Mothia & 2009 \\
\hline Mazara del Vallo & 2008 \\
\hline Guarrato & 2009 \\
\hline Mothia & 2009 \\
\hline Pispisia & 2009 \\
\hline Guarrato & 2009 \\
\hline Guarrato & 2009 \\
\hline Pispisia & 2008 \\
\hline Tre Fontane & 2009 \\
\hline Mazara del Vallo & 2009 \\
\hline Pispisia & 2008 \\
\hline
\end{tabular}

FIG. 1. Dendrogram resulting from interdelta analysis of S. cerevisiae strains. 
those estimated on WL. Although in the samples obtained from Musciuleo and Pietra Rinosa vineyards S. cerevisiae was never isolated in both vintages object of analysis, it resulted dominant, alone (in the majority of the vineyards analyzed) or in combination with other species such as H. uvarum, Hanseniaspora opuntie and $L$. thermotolerans, reaching concentrations within 6-8 Log CFU/mL. In general, when S. cerevisiae was not detected, the species dominating the fermentation process were H. uvarum, P. kudriavzevii or C. zemplinina.

Typing of $S$. cerevisiae strains and geographic distribution The 447 isolates belonging to the species $S$. cerevisiae were further genetically characterized. The interdelta analysis was able to separate the isolates in 51 groups, while microsatellite multiplex PCR recognized 44 different groups, showing a lower discriminatory power than the first technique. A dendrogram resulting from the cluster analysis of the 51 interdelta profiles is reported in Fig. 1. Except a few strains found in the same vineyard in a given year (CS136 and CS179; CS338 and CS339) which clustered at high levels ( $>90 \%$ ), no particular similarities were found among strains isolated within the same vineyard. Furthermore, no strain was found in different vineyards or vintages.

The distribution of $S$. cerevisiae strains among the vineyards analyzed (Table 4) was found to be non-homogeneous. When S. cerevisiae was found, the number of strains recognized was between 1 and 12; the sampling from Lago Preola, Madonna Paradiso and Triglia Scaletta sites produced a very low number of strains, on the contrary Mothia, Pispisia and Mazara del Vallo were richer in S. cerevisiae biodiversity with 12,11 and 10 different strains, respectively.

Technological screening of $\boldsymbol{S}$. cerevisiae strains The 51 $S$. cerevisiae strains were screened for their enological characters (Table 5). Thirty-two strains were characterized by a low production of $\mathrm{H}_{2} \mathrm{~S}$ on Biggy agar plates (white-light brown colony) and resistance to high levels of ethanol $(14-16 \% \mathrm{v} / \mathrm{v})$. Moreover, 36 and 48 strains showed growth in presence of high concentrations of KMBS (150-300 mg/L) and $\mathrm{CuSO}_{4}(400-500 \mathrm{mmol} / \mathrm{L})$, respectively. Twenty-eight strains were found to produce low levels of acetic acid. The growth at low temperatures $\left(13^{\circ} \mathrm{C}\right.$ and $\left.17{ }^{\circ} \mathrm{C}\right)$ was positive for 22 strains, whereas all 51 developed in suspension. Only five strains were found to produce more than $2 \mathrm{~mm}$ of foam.

From the previous technological tests, 14 strains were selected and used as starters to ferment grape must at $13^{\circ} \mathrm{C}$ and $17{ }^{\circ} \mathrm{C}$ in presence of $100 \mathrm{mg} / \mathrm{L}$ of KMBS. The results of the fermentation kinetics (Table 6) showed that, in terms of FP, FR and FPu, three strains (CS160, CS165 and CS182) showed better technological aptitudes than control strains.

After fermentation, enzymatic activities were determined as quality parameters (Table 6 ). The above three strains were characterized by optimal $\beta$-glucosidase activity, in particular onto agar plates containing esculin and MUG. However, no S. cerevisiae showed protease activity.

TABLE 4. Geographical and annual distribution of S. cerevisiae strains during spontaneous fermentations.

\begin{tabular}{lccccccc}
\hline Vineyards & \multicolumn{2}{c}{ No. of S. cerevisiae isolates } & & \multicolumn{2}{c}{ No. of S. cerevisiae strains } \\
\cline { 2 - 4 } \cline { 8 - 9 } & 2008 & 2009 & Total & & 2008 & 2009 & Total \\
\hline Guarrato & 28 & 43 & 71 & & 2 & 4 & 6 \\
Lago Preola & - & 31 & 31 & & - & 1 & 1 \\
Madonna paradiso & - & 33 & 33 & & - & 2 & 2 \\
Mazara del Vallo & 26 & 38 & 64 & & 4 & 6 & 10 \\
Mothia & 26 & 46 & 72 & & 3 & 8 & 11 \\
Musciuleo & - & - & - & & - & - & - \\
Pietra Rinosa & - & - & - & & - & - & - \\
Pispisia & 34 & 47 & 81 & & 5 & 7 & 12 \\
Tre Fontane & - & 48 & 48 & & - & 6 & 6 \\
Triglia Scaletta & 47 & - & 47 & & 3 & - & 3 \\
Total & 161 & 286 & 447 & & 18 & 33 & 51 \\
\hline
\end{tabular}

\section{DISCUSSION}

Microbial dynamics are important during long-term fermentation processes, such as wine productions, since the availability of the grapes occurs once a year and an anomalous evolution of the

TABLE 5. Technological screening of $S$. cerevisiae strains.

\begin{tabular}{|c|c|c|c|c|c|c|c|c|c|}
\hline $\begin{array}{l}\text { Strain } \\
\text { code }\end{array}$ & $\mathrm{H}_{2} \mathrm{~S}^{\mathrm{a}}$ & Ethanol $^{\mathrm{b}}$ & $\mathrm{KMBS}^{\mathrm{c}}$ & $\mathrm{CuSO}_{4}{ }^{\mathrm{d}}$ & $\mathrm{CaCO}_{3}{ }^{\mathrm{e}}$ & $13^{\circ} \mathrm{C}^{\mathrm{f}}$ & $17^{\circ} \mathrm{C}^{\mathrm{g}}$ & $\begin{array}{l}\text { Growth } \\
\text { pattern }^{h}\end{array}$ & Foam $^{\mathrm{i}}$ \\
\hline CS71 & 2 & 4 & 6 & 10 & - & + & + & $S$ & F0 \\
\hline CS72 & 4 & 2 & 5 & 9 & - & - & - & $S$ & F0 \\
\hline CS100 & 3 & 3 & 4 & 8 & + & - & - & $S$ & F0 \\
\hline CS127 & 1 & 2 & 5 & 8 & - & - & - & $\mathrm{S}$ & $\mathrm{F} 1$ \\
\hline CS128 & 0 & 4 & 6 & 10 & - & + & + & $S$ & F0 \\
\hline CS129 & 3 & 3 & 4 & 8 & + & + & + & $\mathrm{S}$ & F0 \\
\hline CS133 & 0 & 4 & 6 & 10 & - & + & + & $S$ & F0 \\
\hline CS136 & 1 & 2 & 3 & 8 & + & - & - & $S$ & F0 \\
\hline CS139 & 4 & 3 & 5 & 8 & - & - & - & $S$ & F0 \\
\hline CS148 & 1 & 4 & 5 & 10 & - & + & + & $S$ & F0 \\
\hline CS155 & 1 & 4 & 6 & 10 & - & + & + & $S$ & F0 \\
\hline CS160 & 2 & 4 & 6 & 10 & - & + & + & $S$ & F0 \\
\hline CS162 & 1 & 4 & 6 & 10 & - & + & + & $S$ & F0 \\
\hline CS165 & 0 & 4 & 6 & 10 & - & + & + & $S$ & F0 \\
\hline CS178 & 2 & 1 & 3 & 8 & + & - & - & $S$ & $\mathrm{~F} 1$ \\
\hline CS179 & 4 & 3 & 3 & 9 & - & - & - & $S$ & F0 \\
\hline CS180 & 1 & 4 & 6 & 10 & - & + & + & $S$ & F0 \\
\hline CS182 & 2 & 4 & 6 & 10 & - & + & + & $S$ & F0 \\
\hline CS255 & 4 & 4 & 4 & 9 & - & + & + & $S$ & F0 \\
\hline CS267 & 3 & 3 & 4 & 8 & + & - & - & $S$ & F0 \\
\hline CS274 & 2 & 2 & 3 & 9 & + & - & - & $S$ & F0 \\
\hline CS275 & 4 & 3 & 4 & 10 & + & + & + & $S$ & F0 \\
\hline CS277 & 3 & 1 & 4 & 9 & + & - & - & $S$ & $\mathrm{~F} 1$ \\
\hline CS278 & 4 & 4 & 3 & 8 & - & + & + & $S$ & $\mathrm{~F} 1$ \\
\hline CS289A & 4 & 3 & 4 & 7 & - & - & - & $S$ & F0 \\
\hline CS289B & 4 & 3 & 3 & 8 & + & - & - & $S$ & F0 \\
\hline CS292 & 2 & 3 & 4 & 8 & + & - & - & $S$ & F0 \\
\hline CS295 & 3 & 1 & 3 & 9 & - & - & - & $S$ & F0 \\
\hline CS309 & 4 & 3 & 4 & 8 & - & - & - & S & F0 \\
\hline CS310 & 4 & 4 & 4 & 7 & + & - & - & $S$ & F0 \\
\hline CS311 & 3 & 2 & 5 & 8 & - & - & - & $S$ & F0 \\
\hline CS313 & 3 & 3 & 5 & 9 & + & - & - & S & F0 \\
\hline CS314 & 4 & 2 & 5 & 9 & + & + & + & $\mathrm{S}$ & F0 \\
\hline CS315 & 3 & 1 & 4 & 7 & - & - & - & $\mathrm{S}$ & F0 \\
\hline CS316 & 4 & 2 & 4 & 10 & - & + & + & $S$ & F0 \\
\hline CS317 & 2 & 2 & 3 & 9 & + & - & - & $\mathrm{S}$ & F0 \\
\hline CS318 & 2 & 1 & 3 & 8 & + & - & - & $S$ & F0 \\
\hline CS319 & 3 & 1 & 3 & 9 & + & - & - & $S$ & F0 \\
\hline CS320 & 4 & 2 & 5 & 10 & - & + & + & $S$ & F0 \\
\hline CS321 & 4 & 3 & 4 & 9 & + & + & + & $S$ & F0 \\
\hline CS322 & 3 & 1 & 3 & 8 & - & - & - & $S$ & F0 \\
\hline CS323 & 2 & 2 & 3 & 9 & + & - & - & $S$ & F0 \\
\hline CS325 & 1 & 3 & 4 & 9 & + & - & - & $S$ & F0 \\
\hline CS326 & 1 & 3 & 3 & 8 & + & - & - & $S$ & F0 \\
\hline CS327 & 1 & 2 & 4 & 8 & + & - & - & $\mathrm{S}$ & F0 \\
\hline CS328 & 1 & 2 & 3 & 8 & + & - & - & $S$ & F0 \\
\hline CS329 & 1 & 3 & 5 & 10 & - & + & + & $\mathrm{S}$ & F0 \\
\hline CS331 & 1 & 4 & 6 & 10 & - & + & + & $\mathrm{S}$ & F0 \\
\hline CS332 & 1 & 4 & 3 & 8 & + & - & - & $S$ & $\mathrm{~F} 1$ \\
\hline CS338 & 1 & 4 & 5 & 10 & - & + & + & $S$ & F0 \\
\hline CS339 & 1 & 4 & 5 & 10 & - & + & + & $S$ & F0 \\
\hline
\end{tabular}

a Color of colony on Biggy agar plates: 0, white; 1, beige; 2, light brown; 3, brown; 4, dark brown; 5 , black.

b $0,0 \%(\mathrm{v} / \mathrm{v}) ; 1,10 \%(\mathrm{v} / \mathrm{v}) ; 2,12 \%(\mathrm{v} / \mathrm{v}) ; 3,14 \%(\mathrm{v} / \mathrm{v}) ; 4,16 \%(\mathrm{v} / \mathrm{v})$ of ethanol contained in MESA plates at which strains showed growth.

c $50 \mathrm{mg} / \mathrm{L} ; 2,100 \mathrm{mg} / \mathrm{L} ; 3,150 \mathrm{mg} / \mathrm{L} ; 4,200 \mathrm{mg} / \mathrm{L} ; 5,250 \mathrm{mg} / \mathrm{L} ; 6,300 \mathrm{mg} / \mathrm{L}$ of MBSK contained into MESA plates at which strains showed growth.

d $0,0 \mu \mathrm{M} ; 1,50 \mu \mathrm{M} ; 2,100 \mu \mathrm{M} ; 3,150 \mu \mathrm{M} ; 4,200 \mu \mathrm{M} ; 5,250 \mu \mathrm{M} ; 6,300 \mu \mathrm{M} ; 7$, $350 \mu \mathrm{M} ; 8,400 \mu \mathrm{M} ; 9,450 \mu \mathrm{M} ; 10,500 \mu \mathrm{M}$ of $\mathrm{CuSO}_{4}$ contained into YPD agar plates at which strains showed growth.

e Result of analysis: +, precipitation halo; -, non-precipitation halo on $\mathrm{CaCO}_{3}$ agar plates.

${ }^{\mathrm{f}}$ Result of analysis: +, growth; - , no growth at $13^{\circ} \mathrm{C}$ in YPD broth.

${ }^{g}$ Result of analysis: +, growth; -, no growth at $17^{\circ} \mathrm{C}$ in YPD broth.

${ }^{h} \mathrm{~S}$, suspended growth; F, flocculant growth in YPD broth.

${ }^{i}$ Fo, foaming lower than $2 \mathrm{~mm}$; F1, foaming among 2 and $4 \mathrm{~mm}$; F2, foaming greater than $4 \mathrm{~mm}$. 


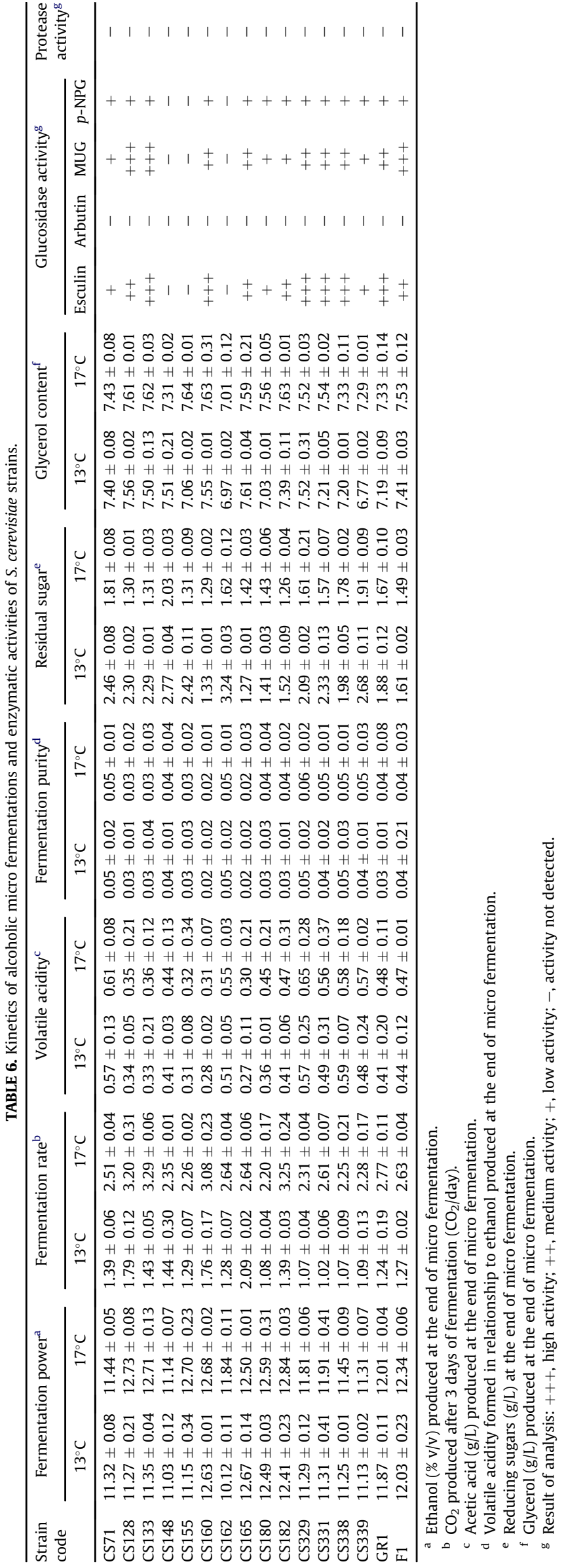

microorganisms in the fermenting musts may determine low quality products and conspicuous economic losses for producers.

In the present work, we pictured the structure of yeast communities present on the grapes of Grillo cultivar, in must and during its steps of spontaneous fermentations, focusing on the technological selection of $S$. cerevisiae strains. Ten vineyards, representing the principal sites of Marsala wine production area, were sampled during two consecutive years (2008 and 2009). Yeast counts reflected a non-homogeneous distribution among sampling sites and vintages, but, in general, the effect of vineyard, year and sample determined significant differences on the concentrations of TY and PS. The finding that the majority of yeasts occurring on grapes did not belong to the Saccharomyces genus is in agreement with previous reports (29).

The process of isolation resulted in the collection of 1144 yeasts. After restriction analysis of 5.8S-ITS rRNA region and 26S rRNA gene, 14 yeast groups were recognized. Only three of them were easily identified at species level, whereas for the other 11 groups, characterized by atypical restriction profiles of 5.8S-ITS, the sequencing of the D1/D2 domain of the 26S rRNA gene was necessary. Atypical polymorphism for this region is not surprising for yeasts, since many authors observed this behavior in several strains (30-32). At the end of the identification process, 14 species belonging to 10 genera (Aureobasidium, Candida, Cryptococcus, Hanseniaspora, Issatchenkia, Lachanceae, Metschnikowia, Pichia, Saccharomyces and Wicherhamomyces) were found.

The yeast communities present on the samples resulted complex. As previously stated by other authors $(29,33)$, NS yeasts were dominant on grapes and in must soon after pressing, while only a few species (H. uvarum, S. cerevisiae, C. zemplinina and P. kudriavzevii) represented the prevailing flora during the stages of fermentation. Although the frequency of the species is generally calculated on the total number of isolates collected from the different vineyards and in the entire period of observation, which may include consecutive vintages $(20,34,35)$, we found this approach arbitrary. The species proportion is unavoidably altered by the isolation process, that is performed randomly. In this study we analyzed the yeast species distribution based on their effective concentrations (Table 3).

$H$. uvarum was the species mainly isolated during fermentation. In some cases it was found at levels of $10^{7}-10^{8} \mathrm{CFU} / \mathrm{mL}$ in both vintages. Its high frequency of isolation at these stages confirms a general behavior observed for other grape varieties $(34,36)$. The distribution of $H$. uvarum in different geographic regions might be linked to the low altitude and high temperature (37), climatic factors that characterize the area of production of Marsala wine. Within Hanseniaspora genus, Hanseniaspora guilliermondii is the species reported to be mainly present in warm climates (35), but in our study it was isolated in a few samples, not above $10^{7} \mathrm{CFU} / \mathrm{mL}$, collected only during 2009 vintage. The species $H$. opuntiae was also isolated. Interestingly, this species was found when $H$. uvarum was absent and its presence was more frequent in the vintage 2009. $H$. opuntiae has been reported to be a member of the grape ecosystem (38) and to dominate the first stages of alcoholic fermentation (39), but no information is available in literature on its presence at the late phases of the process. In this work $H$. opuntiae was detected at approximately $10^{7} \mathrm{CFU} / \mathrm{mL}$ at $3 / 5$ sugar consumption.

Another species isolated at high frequency on grapes and in must soon after pressing was $M$. pulcherrima. This result could be due to the capability of this species to prevail by inhibiting the growth of different yeasts, including S. cerevisiae (40). A. pullulans was also particularly present in these samples, but only in 2009 vintage. Generally, this species has been detected on unripe grape berries (11) and in grape musts $(3,29)$ and Verginer et al. (41) reported its influence in the flavor development of red wines. In the 
present study, strains of this species were isolated only from WL agar plates, even at $10^{6} \mathrm{CFU} / \mathrm{mL}$, showing their susceptibility to the selective conditions of MESA; hence, they do not represent potential wine contaminants. Among the yeast species isolated at low frequency, it is interestingly to note the presence of Cryptococcus flavescens isolated on grapes at $10^{4} \mathrm{CFU} / \mathrm{g}$ in a single vineyard and reported to be isolated on this matrix only once before (34).

The spontaneous fermentations were then dominated by $H$. uvarum, S. cerevisiae, C. zemplinina and P. kudriavzevii. Despite the selective conditions of fermentation, NS populations reached levels of concentration comparable to the PS load until the end of fermentation. Several researchers have focussed on the positive influence of NS yeasts emphasizing their potential application as starters in wine productions $(7,25)$. Furthermore, the use of Hanseniaspora spp. in combination with $S$. cerevisiae has been reported to contribute positively to the complexity and aroma of wine $(6,42)$. This may be due to the capability of these yeasts, e.g., H. uvarum strains, to secrete several enzymes, such as $\beta$-glucosidase and proteases, that could contribute to the expression of varietal aroma of grapevine (37,43). C. zemplinina was also isolated in several samples at high concentrations (till $10^{7}-10^{8} \mathrm{CFU} / \mathrm{mL}$ ). These strains could represent an important source of starters to be employed for mixed fermentations with $S$. cerevisiae, since their interaction was demonstrated to increase the fermentation kinetics of grape must (44). Moreover, some C. zemplinina strains are osmotolerants, producers of low concentration of acetic acid and high amounts of glycerol from sugars (45) and may found application to reduce the ethanol content of wines produced by grape musts characterized by high sugar content, such as those produced in the Marsala area. Regarding P. kudriavzevii, it is usually detected on grapes (34) and in the early stages of alcoholic fermentation (46), thus, its finding at the latest stages of fermentation needs further investigation.

Yeast numbers and species recovered in this study are consistent with the presence of rotten berries hidden in undamaged clusters. The influence of rotten grapes on yeast species diversity is a wellknown phenomenon (47). Hence, grape sampling plays a defining role in the structure of the yeast populations estimated (48).

$S$. cerevisiae strains selected from indigenous populations of a given area might drive the alcoholic fermentation better than commercial starters (49). Due to their enological importance, all $S$. cerevisiae cultures isolated in this work were investigated at strain level. Cluster analysis recognized 51 strains and showed that no common pattern was found among strains isolated from different vineyards or vintages. Many authors claimed that autochthonous yeasts are linked to a specific area $(49,50)$ and stable in consecutive years (50), but for others, the occurrence of strains in the vineyards is only temporary (51).

Based on their technological properties, especially on their ethanol resistance, $14 \mathrm{~S}$. cerevisiae strains were selected and tested as starters in Grillo grape must. Among them, only two couples of strains (CS133-CS165 and CS338-CS339) found in the same vineyard in the same year shared a certain genetic similarity, but no other strain was found in different vineyards or vintages. Three strains (CS160, CS165 and CS182) were characterized by a relevant FP, a capacity of paramount importance in this type of wine, since a high rate of sugar consumption is mandatory.

The main conclusions of this work are: yeast populations analyzed in 10 vineyards located in the area of Marsala DOC wine, which have never been explored before, showed some differences in species composition and concentration levels between the two consecutive years (2008 and 2009) object of study; H. uvarum, C. zemplinina and, interestingly, P. kudriavzevii were detected in place of or at comparable levels of $S$. cerevisiae in the stages of fermentation characterized by high ethanol concentration; 14 autochthonous $S$. cerevisiae strains displayed a technological potential to drive the fermentation of must into wine. The technological investigation of NS isolates is being prepared in order to design mixed strain starters for the preservation of the typicality of the wines obtained with Grillo cultivar.

\section{References}

1. Subden, R. E.: Current developments in wine yeasts, Crit. Rev. Biotechnol., 5, 49-65 (1987).

2. Valero, E., Schuller, D., Cambon, B., Casal, M., and Dequin, S.: Dissemination and survival of commercial wine yeast in the vineyard: a large-scale, threeyears study, FEMS Yeast Res., 5, 959-969 (2005).

3. Francesca, N., Chiurazzi, M., Romano, R., Aponte, M., Settanni, L., and Moschetti, G.: Indigenous yeast communities in the environment of "Rovello Bianco" grape variety and their use in commercial white wine fermentation, World J. Microbiol. Biotechnol., 26, 337-351 (2010).

4. Martinez, J., Millan, C., and Ortega, J. M.: Growth of natural flora during the fermentation of inoculated musts from 'Pedro Ximenez' grapes, S. Afr. J. Enol. Vitic., 10, 31-35 (1989).

5. Mora, J., Barbas, J. I., and Mulet, A.: Growth of yeast species during the fermentation of musts inoculated with Kluyveromyces thermotolerans and Saccharomyces cerevisiae, Am. J. Enol. Vitic., 41, 156-159 (1990).

6. Ciani, M., Beco, L., and Comitini, F.: Fermentation behaviour and metabolic interactions of multistarter wine yeast fermentations, Int. J. Food Microbiol., 108, 239-245 (2006).

7. Anfang, N., Brajkovich, M., and Goddard, M. R.: Co-fermentation with Pichia kluyveri increases varietal thiol concentrations in Sauvignon Blanc, Aust. J. Grape Wine Res., 15, 1-8 (2009).

8. Soden, A., Francis, I. L., Oackey, H., and Henschke, P. A.: Effects of co-fermentation with Candida stellata and Saccharomyces cerevisiae on the aroma and composition of Chardonnay wine, Aust. J. Grape Wine Res., 6, $21-30$ (2000).

9. Charoenchai, C., Fleet, G. H., Henschke, P. A., and Todd, B. E. N.: Screening of non-Saccharomyces wine yeasts for the presence of extracellular hydrolytic enzymes, Aust. J. Grape Wine Res., 3, 2-8 (1997).

10. Pretorius, I., van der Westhuizen, T., and Augustyn, $\mathbf{0}$.: Yeast biodiversity in vineyard and wineries and its importance to the south African wine industry. A review, S. Afr. J. Enol. Vitic., 20, 61-74 (1999).

11. Renouf, V., Claisse, O., and Lonvaud-Funel, A.: Understanding the microbial ecosystem on the grape berry surface through numeration and identification of yeast and bacteria, Aust. J. Grape Wine Res., 11, 316-327 (2005).

12. Esteve-Zarzoso, B., Belloch, C., Uruburu, F., and Querol, A.: Identification of yeasts by RFLP analysis of the 5.8S rRNA gene and the two ribosomal internal transcribed spacers, Int. J. Syst. Bacteriol., 49, 329-337 (1999).

13. Baleiras-Couto, M. M., Reizinho, R. G., and Duarte, F. L.: Partial 26S rDNA restriction analysis as a tool to characterize non-Saccharomyces yeasts present during red wine fermentations, Int. J. Food Microbiol., 102, 49-56 (2005).

14. O'Donnell, K.: Fusarium and its near relatives, pp. 225-233, in: Reynolds, D. R. and Taylor, J. W. (Eds.), The fungal anamorph: mitotic, meiotic and pleomorphic speciation in fungal systematic. CAB International, Wallingford (1993).

15. Legras, J. L. and Karst, F.: Optimisation of interdelta analysis for Saccharomyces cerevisiae strain characterization, FEMS Microbiol. Lett., 221, 249-255 (2003).

16. Vaudano, E. and Garcia-Moruno, E.: Discrimination of Saccharomyces cerevisiae wine strains using microsatellite multiplex PCR and band pattern analysis, Food Microbiol., 25, 56-64 (2008).

17. Jiranek, V., Langridge, P., and Henschke, P. A.: Validation of bismuth-containing indicator media for predicting $\mathrm{H}_{2} \mathrm{~S}$ producing potential of Saccharomyces cerevisiae wine yeasts under enological conditions, Am. J. Enol. Vitic., 46, 269-273 (1995).

18. Fiore, C., Arrizon, J., Gschaedler, A., Flores, J., and Romano, P.: Comparison between yeasts from grape and agave musts for traits of technological interest, World J. Microbiol. Biotechnol., 21, 1141-1147 (2005).

19. Caridi, A., Cufari, A., and Ramondino, D.: Isolation and clonal pre-selection of enological Saccharomyces, J. Gen. Appl. Microbiol., 48, 261-267 (2002).

20. Di Maio, S., Polizzotto, G., Di Gangi, E., Foresta, G., Genna, G., Verzera, A., Scacco, A., Amore, G., and Oliva, D.: Biodiversity of indigenous Saccharomyces populations from old wineries of south-eastern Sicily (Italy): preservation and economic potential, PLoS One, 7, e30428 (2012).

21. Regodón, J. A., Peréz, F., Valdé, M. E., De Miguel, C., and Ramírez, M.: A simple and effective procedure for selection of wine yeast strains, Food Microbiol., 14, 247-254 (1997).

22. Hernández, L. F., Espinosa, J. C., Fernández-González, M., and Briones, A.: $\beta$-Glucosidase activity in a Saccharomyces cerevisiae wine strain, Int. J. Food Microbiol., 80, 171-176 (2003).

23. Bilinsky, C. A., Russell, I., and Stewart, G. G.: Applicability of yeast extracellular proteinases in brewing: physiological and biochemical aspects, Appl. Environ. Microbiol., 53, 495-499 (1987).

24. Ciani, M. and Rosini, G.: Definizione dell'indice di moltiplicazione della $\mathrm{CO}_{2}$ nella valutazione, per via ponderale, della capacità alcoligena di un lievito, Annali della Facoltà di Agraria di Perugia, 41, 753-762 (1987) (in Italian). 
25. Ciani, M. and Maccarelli, F.: Oenological properties of non-Saccharomyces yeasts associated with winemaking, World J. Microbiol. Biotechnol., 14, 199-203 (1998).

26. OIV: Recueil des méthodes internationales d'analyse des vins et des moûts. Organisation Interantionel de la Vigne e du Vin, Paris (2010) (in French).

27. Cordero-Bueso, G., Arroyo, T., Serrano, A., Tello, J., Aporta, I., Vélez, M. D., and Valero, E.: Influence of the farming system and vine variety on yeast communities associated with grape berries, Int. J. Food Microbiol., 145, 132-139 (2011).

28. Muccilli, S., Caggia, C., Randazzo, C. L., and Restuccia, C.: Yeast dynamics during the fermentation of brined green olives treated in the field with kaolin and Bordeaux mixture to control the olive fruit fly, Int. J. Food Microbiol., 148, 15-22 (2011).

29. Sabaté, J., Cano, J., Esteve-Zarzoso, B., and Guillamón, J. M.: Isolation and identification of yeasts associated with vineyard and winery by RFLP analysis of ribosomal genes and mitochondrial DNA, Microbiol. Res., 157, 267-274 (2002).

30. Kurtzman, C. P. and Robnett, C. J.: Phylogenetic relationships among yeast of the 'Saccharomyces complex' determined from multigene sequence analyses, FEMS Yeast Res., 3, 417-432 (2003).

31. Tofalo, R., Chaves-López, C., Di Fabio, F., Schirone, M., Felis, G. E., Torriani, S., Paparella, A., and Suzzi, G.: Molecular identification and osmotolerant profile of wine yeasts that ferment a high sugar grape must, Int. J. Food Microbiol., 130, 179-187 (2009).

32. Francesca, N., Canale, D. E., Settanni, L., and Moschetti, G.: Dissemination of wine-related yeasts by migratory birds, Environ. Microbiol. Rep., 4, 105-112 (2012).

33. González, S. S., Barrio, E., and Querol, A.: Molecular identification and characterization of wine yeasts isolated from Tenerife (Canary Island, Spain), J. Appl. Microbiol., 102, 1018-1025 (2007).

34. Li, S. S., Cheng, C., Li, Z., Chen, J.-Y., Yan, B., Han, B.-Z., and Reeves, M.: Yeast species associated with wine grapes in China, Int. J. Food Microbiol., 138, 85-90 (2010).

35. Romancino, D. P., Di Maio, S., Muriella, R., and Oliva, D.: Analysis of nonSaccharomyces yeast populations isolated from grape musts from Sicily (Italy), J. Appl. Microbiol., 105, 2248-2254 (2008).

36. Raspor, P., Milek, D. M., Polanc, J., Možina, S. S., and Čadež, N.: Yeasts isolated from three varieties of grapes cultivated in different locations of the Dolenjska vine-growing region, Slovenia, Int. J. Food Microbiol., 109, 97-102 (2006).

37. Jolly, N. P., Augustyn, O. P. H., and Pretorius, I. S.: The role and use of nonSaccharomyces yeasts in wine production, S. Afr. J. Enol. Vitic., 27, 15-39 (2006).
38. Nisiotou, A. A. and Nychas, G.-J. N.: Yeast populations residing on healthy or Botrytis-infected grapes from a vineyard in Attica, Greece, Appl. Environ. Microbiol., 73, 2765-2768 (2007).

39. Bovo, B., Andrighetto, C., Carlot, M., Corich, V., Lombardi, A., and Giacomini, A.: Yeast population dynamics during pilot-scale storage of grape marcs for the production of Grappa, a traditional Italian alcoholic beverage, Int J. Food Microbiol., 129, 221-228 (2009).

40. Nguyen, H. V. and Panon, G.: The yeast Metschnikowia pulcherrima has an inhibitory effect against various yeast species, Sci. Aliments, 18, 515-526 (1998).

41. Verginer, M., Leitner, E., and Berg, G.: Production of volatile metabolites by grape-associated microorganisms, J. Agric. Food Chem., 58, 8344-8350 (2010).

42. Moreira, N., Mendes, F., Guedes de Pinho, P., Hogg, T., and Vasconcelos, I.: Heavy sulphur compounds, higher alcohols and esters production profile of Hanseniaspora uvarum and Hanseniaspora guilliermondii grown as pure and mixed cultures in grape must, Int. J. Food Microbiol., 124, 231-238 (2008).

43. Zott, K., Miot-Sertier, C., Claisse, O., Lonvaud-Funel, A., and MasneufPomarede, I.: Dynamics and diversity of non-Saccharomyces yeasts during the early stages in winemaking, Int. J. Food Microbiol., 125, 197-203 (2008).

44. Tofalo, R., Schirone, M., Torriani, S., Rantsiou, K., Cocolin, L., Perpetuini, G., and Suzzi, G.: Diversity of Candida zemplinina strains from grapes and Italian wines, Food Microbiol., 29, 18-26 (2012)

45. Sipiczki, M., Ciani, M., and Csoma, H.: Taxonomic reclassification of Candida stellata DBVPG 3827, Folia Microbiol., 50, 494-498 (2005).

46. Di Maro, E., Ercolini, D., and Coppola, S.: Yeast dynamics during spontaneous wine fermentation of the Catalanesca grape, Int. J. Food Microbiol., 117, 201-210 (2007).

47. Barata, A., Malfeito-Ferreira, M., and Loureiro, V.: The microbial ecology of wine grape berries, Int. J. Food Microbiol., 153, 243-259 (2012).

48. Barata, A., Malfeito-Ferreira, M., and Loureiro, V.: Changes in sour rotten grape berry microbiota during ripening and wine fermentation, Int. J. Food Microbiol., 154, 152-161 (2012).

49. Lopes, C. A., van Broock, M., Querol, A., and Caballero, A. C.: Saccharomyces cerevisiae wine yeast populations in a cold region in Argentinean Patagonia. A study at different fermentation scales, J. Appl. Microbiol., 93, 608-615 (2002).

50. Schuller, D., Alves, H., Dequin, S., and Casal, M.: Ecological survey of Saccharomyces strains from vineyards in the Vinho Verde Region of Portugal, FEMS Microbiol. Ecol., 51, 167-177 (2005).

51. Prakitchaiwattana, C. J., Fleet, G. H., and Heard, G. M.: Application and evaluation of denaturing gradient gel electrophoresis to analyse the yeast ecology of wine grapes, FEMS Yeast Res., 4, 856-877 (2004). 